\title{
Plasma glucose levels in term infants who are appropriate size for gestation and exclusively breast fed
}

\author{
K K Diwakar, M V Sasidhar
}

Arch Dis Child Fetal Neonatal Ed 2002;87:F46-F48

See end of article for authors' affiliations

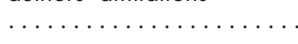

Correspondence to: Professor Diwakar,

Neonatal Division, Department of Pediatrics, Kasturba Medical College,

Manipal, Karnataka -

576119, India

kk.diwakar@

kmc.manipal.edu

Accepted

29 November 2001

\begin{abstract}
Aim: To evaluate the plasma glucose levels in normal, term infants who were appropriate size for gestational age (AGA) and exclusively breast fed, and to assess the influence of parity of the mother, mode of delivery, and time of feed on the glucose levels.

Method: A total of 200 healthy, term, AGA infants were longitudinally evaluated at 3, 6, 24, and 72 hours of life. Plasma glucose was estimated from heel prick capillary samples. The influence of mode of delivery, parity, and interval between feeds on plasma glucose was analysed.

Results: There was no significant difference between the plasma glucose levels of the cohorts at any of the sampling time points. Parity, mode of delivery, and time since the last feed did not affect plasma glucose. Satisfactory glucose levels were maintained even when infants remained unfed up to 6 hours of age. Infants with plasma glucose concentrations less than $2.2 \mathrm{mmol} / \mathrm{l}$ at 3 hours of age were more likely to have low sugar concentration $(<2.5 \mathrm{mmol} / \mathrm{l})$ at 72 hours $(R R=6.55,95 \%$ confidence interval 3.93 to 10.92 ).

Conclusions: A term, breast fed infant may have its own distinct plasma glucose levels, showing no significant variation between 3 and 72 hours of age. Plasma glucose levels are satisfactorily maintained in normal term infants without resort to prelacteal feeds. Mode of delivery, parity of the mother, and interval between feeds did not influence plasma glucose. Biochemical thresholds for hypoglycaemia do not seem to be of practical importance in asymptomatic, normal, term, breast fed infants.
\end{abstract}

\section{MATERIALS AND METHODS}

Two hundred normal, term, appropriate for gestational age (AGA) infants delivered at the Kasturba Hospital, Manipal were included in the study. We defined "term" as delivered at 37-42 weeks gestation, based on maternal dates. Birth weight was defined as AGA on the basis of Usher's chart. ${ }^{5}$ The mean birth weight was $2650 \mathrm{~g}$ (range 2300-4290), with a male to female ratio of 104:96. Ninety seven infants were born to primiparous mothers, and 103 were born to multipara. A total of 130 of the infants were born vaginally, and 70 were delivered by lower segment caesarean section. Infants with respiratory distress, perinatal asphyxia, meconium aspiration syndrome, or polycythaemia (capillary packed cell volume $>70$ ), infants receiving antibiotics, and those born to mothers with a history of hypertension, anaemia, or diabetes mellitus were excluded from the study.

All infants were exclusively breast fed ad libitum, as per hospital policy. Infants delivered by caesarian section were started on breast feeds as soon as the mother was transferred to the ward. No prelacteal feeds were given. Delays in transfer provided us with ethically acceptable "unfed"controls.

Capillary blood was collected by heel prick, centrifuged at $12000 \mathrm{rpm}$ for 10 minutes, and the plasma glucose evaluated within 15 minutes by the glucose oxidase method (Hitachi autoanalyser 902 ) for each infant at 3 hours of age $( \pm 30 \mathrm{~min}-$ utes). These cohorts were followed up and their plasma glucose further assessed at 6, 24, and 72 hours of age (give or take 30 minutes at each age). The 72 hour sample often coincided with other routine investigations such as serum bilirubin. We chose these time points in order to study the most vulnerable period of glucose homoeostasis, while ensuring minimum trauma to the infant, albeit with consent.

Plasma glucose concentrations were analysed with regard to distribution, variation with age, mode of delivery, parity of the mother, and the time since the last feed. Concentrations less than $2.2 \mathrm{mmol} / \mathrm{l}$ at and before 24 hours and below 2.5 $\mathrm{mmol} / \mathrm{l}$ at 72 hours were considered low $^{6}$ for the purpose of analysis. Lethargy, jitteriness, and convulsions, alleviated by a bolus of intravenous glucose, were considered to be clinical signs of hypoglycaemia. Infants were considered asymptomatic if low plasma glucose ${ }^{6}$ concentrations were not associated with clinical signs.

Infants found to have low glucose ${ }^{6}$ were clinically reexamined, given an additional breast feed, and plasma glucose reassessed after 30 minutes. We planned to exclude from further evaluation infants who continued to be "hypoglycaemic" despite these additional feeds and to manage them according to the standard protocol for asymptomatic hypoglycaemia.

Informed consent was obtained from the parents.

Paired and unpaired Student's $t$ test, analysis of variance, and $\chi^{2}$ tests were applied where necessary for statistical analysis using the SPSS (version 7.5 for Windows) statistical package.

\section{RESULTS}

Plasma glucose was serially determined in each of the 200 infants at 3, 6, 24, and 72 hours of age. In 112 of the 800 samples thus analysed, glucose levels were "low". ${ }^{6}$ Thirty infants 
Table 1 Plasma glucose concentrations $(\mathrm{mmol} / \mathrm{l})$ in term, appropriate size for gestation, breast fed infants at four different ages

\begin{tabular}{llllll}
\hline $\begin{array}{l}\text { Age } \\
\text { (hours) }\end{array}$ & Mean (SD) & Median & Range & $\begin{array}{l}\text { Interquartile } \\
\text { range }\end{array}$ & SEM \\
\hline 3 & $3(1.05)$ & 2.8 & $1.4-8.3$ & $2.3-3.3$ & 0.07 \\
6 & $2.95(0.75)$ & 2.8 & $1.6-5.4$ & $2.4-3.3$ & 0.05 \\
24 & $2.89(0.79)$ & 2.9 & $1.3-7.6$ & $2.6-3.3$ & 0.06 \\
72 & $3(0.79)$ & 2.8 & $1.4-7.1$ & $2.6-3.3$ & 0.06 \\
\hline
\end{tabular}

Repeated analysis of variance, $p=0.19$.

Table 2 Comparison of plasma glucose $(\mathrm{mmol} / \mathrm{l})$ in infants born to primiparous ( $n=97)$ and multiparous $(n=103)$ women

\begin{tabular}{lllll}
\hline $\begin{array}{l}\text { Age } \\
\text { (hours) }\end{array}$ & Parity & Mean (SD) & SE & p Value \\
\hline 3 & Primi & $2.86(0.85)$ & 0.09 & \\
& Multi & $3.1(1.21)$ & 0.12 & 0.07 \\
6 & Primi & $2.97(0.79)$ & 0.08 & \\
& Multi & $2.96(0.73)$ & 0.07 & 0.924 \\
24 & Primi & $2.85(0.73)$ & 0.07 & \\
& Multi & $2.93(0.86)$ & 0.08 & 0.44 \\
72 & Primi & $2.99(0.86)$ & 0.09 & \\
& Multi & $3.01(0.75)$ & 0.07 & 0.897 \\
\hline
\end{tabular}

Statistical analysis was by the unpaired $t$ test.

Table 3 Comparison of plasma glucose $(\mathrm{mmol} / \mathrm{l})$ in infants born by caesarean section $(n=70)$ and those delivered vaginally $(n=130)$

\begin{tabular}{lllll}
\hline $\begin{array}{l}\text { Age } \\
\text { (hours) }\end{array}$ & Mode of delivery & Mean (SD) & SE & p Value \\
\hline 3 & Caesarean & $3.01(1.09)$ & 0.131 & \\
& Vaginal & $3(1.03)$ & 0.090 & 0.91 \\
6 & Caesarean & $2.88(0.695)$ & 0.083 & \\
& Vaginal & $3(0.79)$ & 0.069 & 0.25 \\
24 & Caesarean & $2.83(0.73)$ & 0.087 & \\
& Vaginal & $2.92(0.8)$ & 0.073 & 0.44 \\
72 & Caesarean & $2.99(0.98)$ & 0.117 & \\
& Vaginal & $3(0.68)$ & 0.06 & 0.86 \\
\hline
\end{tabular}

Statistical analysis was by the unpaired $t$ test.

showed low plasma glucose at one of the four time points, 21 had two low values, eight had three, and four infants continued to show low values at all four time points. No infant had symptomatic or persistent hypoglycaemia requiring intervention. All infants were asymptomatic and attained euglycaemic levels ${ }^{6}$ after an additional feed.

Despite the wide range of glucose concentrations at each time point, the median and interquartile values continued to be similar. Repeated analysis of variance showed no significant difference between plasma glucose levels at 3, 6, 24, or 72 hours $(\mathrm{p}=0.19)($ table 1$)$.

Parity of the mother (table 2) and the mode of delivery (table 3) did not affect the plasma glucose levels of the cohorts.

Delays in transferring mothers to the postnatal ward resulted in 58 of the 70 infants delivered by caesarean section remaining unfed for their first 3 hours, with 32 of them being fed only after 6 hours. We compared the plasma glucose levels of these "unfed" infants with those born to multiparous mothers and fed within 30 minutes before the glucose estimation (table 4). These groups were selected to compare the "unfed" infant with those assured of satisfactory breast
Table 4 Comparison of plasma glucose $(\mathrm{mmol} / \mathrm{l})$ in "unfed" infants with recently fed infants of multiparous mothers

\begin{tabular}{lllll}
\hline Feed status & $\mathrm{n}$ & Mean (SD) & $\mathrm{SE}$ & $\mathrm{p}$ Value \\
\hline 3 hours of age & & & & \\
$\quad$ Unfed & 58 & $2.97(1.07)$ & 0.142 & \\
$\quad$ Recently fed & 45 & $3.23(1.37)$ & 0.2 & 0.28 \\
6 hours of age & & & & \\
$\quad$ Unfed & 32 & $2.9(0.76)$ & 0.135 & \\
$\quad$ Recently fed & 72 & $3(0.71)$ & 0.084 & 0.45 \\
\hline
\end{tabular}

Statistical analysis was by the unpaired $t$ test.

Table 5 Plasma glucose $(\mathrm{mmol} / \mathrm{l})$ levels in relation to time since the last feed

\begin{tabular}{lllll}
\hline Time since last feed & $3 \mathrm{~h}$ & $6 \mathrm{~h}$ & $24 \mathrm{~h}$ & $72 \mathrm{~h}$ \\
\hline Unfed & & & & \\
$\mathrm{n}$ & 58 & 32 & 0 & 0 \\
Mean & 2.97 & 2.89 & & \\
$\mathrm{SD}$ & 1.08 & 0.76 & & \\
$\mathrm{SE}$ & 0.142 & 0.761 & & \\
$<30$ min & & & & \\
$\mathrm{n}$ & 93 & 139 & 154 & 167 \\
Mean & 3.02 & 2.96 & 2.89 & 2.98 \\
SD & 1.12 & 0.71 & 0.86 & 0.82 \\
SE & 0.116 & 0.06 & 0.069 & 0.636 \\
$30-60$ min & & & & \\
$\mathrm{n}$ & 44 & 24 & 37 & 31 \\
Mean & 3 & 3.12 & 3 & 3 \\
SD & 0.92 & 1 & 0.47 & 0.67 \\
SE & 0.139 & 0.21 & 0.078 & 0.120 \\
$>60$ min & & & & \\
$\mathrm{n}$ & 5 & 5 & 9 & 2 \\
Mean & 3.08 & 2.55 & 2.45 & 2.72 \\
SD & 0.75 & 0.38 & 0.64 & 0.71 \\
SE & 0.337 & 0.169 & 0.213 & 0.5 \\
$\mathrm{P}$ Value & 0.992 & 0.413 & 0.17 & 0.868 \\
\hline
\end{tabular}

Statistical analysis by one way analysis of variance.

Table 6 Plasma glucose distribution at sampling times $(n=200)$

\begin{tabular}{lllll}
\hline Plasma glucose $(\mathrm{mmol} / \mathrm{l})$ & $3 \mathrm{~h}$ & $6 \mathrm{~h}$ & $24 \mathrm{~h}$ & $72 \mathrm{~h}$ \\
\hline$<1.39$ & 0 & 0 & 1 & 0 \\
$1.39-1.6$ & 9 & 2 & 7 & 9 \\
$1.66-1.89$ & 7 & 8 & 8 & 2 \\
$1.94-2.16$ & 17 & 10 & 4 & 9 \\
$2.22-2.44$ & 28 & 34 & 23 & 19 \\
$2.5-2.71$ & 35 & 37 & 46 & 39 \\
$>2.71$ & 104 & 109 & 101 & 122 \\
\hline
\end{tabular}

feeds. Remaining unfed did not seem to significantly influence plasma glucose levels at 3 and 6 hours of age $(p=0.17$, $\mathrm{p}=0.48$ respectively).

We analysed plasma glucose in relation to the time since the last breast feed. Infants were grouped as $(a)$ unfed since birth, (b) glucose estimated within 30 minutes of a feed, (c) glucose estimated within 30-60 minutes of a feed and (d) glucose estimated more than 60 minutes after a feed. One way analysis of variance did not show any significant difference between these groups (table 5).

Thirty three infants had low glucose concentrations ${ }^{6}$ at 3 hours, 20 at 6 hours, 20 at 24 hours, and 39 at 72 hours of age (table 6). The usefulness of early plasma glucose levels in predicting the subsequent glycaemic status of the neonate was 
evaluated by comparing the 3 hour and 6 hour plasma glucose levels with those at 24 and 72 hours. Of the 33 infants with low 3 hour plasma glucose levels, 12 showed low glucose levels at 24 hours of life ( $\mathrm{RR}=3.37 ; 95 \%$ confidence interval $(\mathrm{CI})$ 1.80 to 6.32 ; Fisher's test of exact probability $p=0.0007$ ), and 22 had low glucose levels at 72 hours ( $R R=6.55 ; 95 \%$ CI 3.93 to $10.92 ; \mathrm{p}<0.00001$ ).

Of the 20 infants with low plasma glucose at 6 hours of age, seven had low glucose at 24 hours ( $R R=2.74 ; 95 \%$ CI 1.35 to $5.56)$, and 14 had low glucose at 72 hours of life $(R R=5.04$; 95\% CI 3.17 to 8.01 ).

\section{DISCUSSION}

Interpreting blood glucose levels of infants continues to be a challenge for paediatricians. The cross sectional pattern of studies, the role of formula feeds, and patient characteristics such as growth retardation and gestation have clouded the interpretation in most studies. Therefore we attempted to minimise variations resulting from study design and patient characteristics. This longitudinal study of 200 exclusively breast fed, full term, AGA infants shows that there is no significant variation in their plasma glucose levels from 3 to 72 hours of life (table 1 ; repeated analysis of variance $p=0.19$ ). A similar observation was made by Srinivasan $e^{~} a l^{6}$ in formula fed infants. It must, however, be mentioned here that the present study did not evaluate plasma glucose earlier than 3 hours. Unlike previous observations in cross sectional studies, ${ }^{47}$ we did not observe lower sugar levels on the lst day of life.

There was no significant difference in plasma glucose levels of infants delivered vaginally and those delivered by caesarean section, nor between infants of primiparous and multiparous mothers.

The interval between feeds has previously been noted as a major determinant of blood glucose levels. ${ }^{7}$ However, these authors commented that "prolonged intervals between feeds, up to eight hours were not associated with excessively low blood glucose concentrations". We found that time since the last feed did not correlate significantly with plasma glucose in infants breast fed ad libitum (table 5). As all the infants older than 6 hours in our study population were fed every one and a half to two hours, longer intervals between feeds could not be evaluated at the 24 hour and 72 hour time points. We observed that glucose levels were maintained despite infants remaining unfed for the first 6 hours of life (table 4). These values were comparable to the plasma glucose levels of infants of multiparous mothers, sampled within 30 minutes of breast feeding. This will be of solace to primiparous mothers and women who are keen to exclusively breast feed their babies but worried about the "inadequacy" of lactation during the first day. It is also obvious that prelacteal feeds are not needed to tide over this period of "inadequate lactation".

A satisfactory glucose homoeostasis mechanism seems to function in the normal term infant. In the few who had "low plasma glucose levels" ${ }^{\prime \prime}$ at the time of sampling, an immediate breast feed restored normality. ${ }^{8}$ We consider that the interplay of metabolic adaptations ${ }^{79^{10}}$ ensures appropriate glucose homoeostasis to satisfy the energy requirements of term, breast fed infants.

Defining "normal" values for plasma glucose continues to be difficult. Our patients did not show any significant difference in plasma glucose at any of the assessment time points, implying that an infant may have its own distinctive levels of plasma glucose, influenced by its individual patterns of metabolic adaptation. Infants with "low" plasma glucose levels at 3 hours of life were therefore found to have "low" glucose at 72 hours of life ( $R R=6.55,95 \%$ CI 3.93 to 10.92 ).

None of the infants who had "low" plasma glucose values (table 6) at the specific sampling times showed any clinical signs of hypoglycaemia. However, the glucose levels in all increased immediately after a breast feed. The increased frequency of feeding seen in exclusively breast fed infants ensures that plasma glucose levels do not remain low for prolonged periods. The ability of the neonatal brain to use alternative fuels such as ketone bodies or lactate for oxidative metabolism ${ }^{10}$ may ensure that the infant is "symptom free" during these transient periods of reduced plasma glucose. Long term prospective studies to evaluate the effect of "lower" glucose levels in apparently normal term infants are necessary, before any firm recommendations are made. Until then, we believe that, in the absence of risk factors, it is best to leave the term, breast feeding infant alone and not embark on treatments based on biochemically determined values.

\section{Authors' affiliations \\ K K Diwakar, M V Sasidhar, Department of Pediatrics, Kasturba}

Medical College, Manipal, Karnataka, India

\section{REFERENCES}

1 Koh THHG, Eyre JA, Aynsley-Green A. Neonatal hypoglycemia: the controversy regarding definition. Arch Dis Child 1988:63:1386-98.

2 Sexson WR. Incidence of neonatal hypoglycemia: a matter of definition. J Pediatr 1984;105:149-50.

3 Cornblath M, Schwartz R, Aynsley-Green A, et al. Hypoglycemia in infancy: the need for a rational definition (Ciba Foundation Discussion Meeting). Pediatrics 1990;85:834-7.

4 Hoseth E, Joergensen A, Ebbensen F, et al. Blood glucose levels in a population of healthy breast fed term infants of appropriate size for gestational age. Arch Dis Child Fetal Neonatal Ed 2000;83:F117-19.

5 Usher R, Maclean F. Intrauterine growth of liveborn Caucasian infants at sea level: standard obtained in 7 dimensions of infant born between 25 and 44 weeks of gestation. J Pediatr 1969;74:901.

6 Srinivasan G, Pildes RS, Cattamanchi G, et al. Plasma glucose values in normal neonates: a new look. J Pediatr 1986;109:1 14-17.

7 Hawden JM, Ward Platt MP, Anysley-Green A. Patterns of metabolic adaptation for preterm and term infants in the first neonatal week. Arch Dis Childhood 1992;67:357-65.

8 Heck LJ, Errenberg A. Serum glucose levels in term neonates during the first 48 hours of life. J Pediatr 1987;1 10:119-22.

9 Eidelman Al. Pediatr Clin North Am 2001:48:377-87.

10 Cornblath M, Hawden JM, William AF, et al. Controversies regarding definition of neonatal hypoglycemia: suggested operational thresholds. Pediatrics 2000;105: 1141-5. 